\title{
Unsupervised Extrinsic Parameters Calibration for Multi-beam LIDARs
}

\author{
Zhu Zhu \\ Department of Information Science and Electronic \\ Engineering, Zhejiang University \\ Hangzhou, China \\ zachary.zhu@yahoo.cn
}

\author{
Jilin Liu \\ Zhejiang Provincial Key Laboratory of Information \\ Network Technology, Zhejiang University \\ Department of Information Science and Electronic \\ Engineering, Zhejiang University, \\ Hangzhou, China \\ liujl@zju.edu.cn
}

\begin{abstract}
In this paper we present a technique of unsupervised extrinsic parameters calibration for a multi-beam LIDAR scanner. The proposed method decomposes extrinsic parameter calibration procedure into two parts: computing rotation matrix caused by pitch angle and roll angle through ground plane estimation; computing rotation matrix caused by navigation angle through matching pole-like obstacles in a series of poses. Experiment results demonstrate the efficacy of the calibration technique.
\end{abstract}

Keywords-component; unsupervised; calibration; extrinsic; decompose; multi-beam lidar;

\section{INTRODUCTION}

Light Detection and Ranging (LIDAR) sensors are important parts of robot systems. Traditional LIDAR sensors are equipped with only one rotating beam. However, with the progress of science and technology, applying multi-beam LIDAR sensors which are often referred as 3D LIDAR sensors configured with many simultaneous rotating beams at varying angles become a trend in robot navigation. One of these systems is the Velodyne HDL-64E S2(Fig. 1): it consists of 64 lasers located on a spinning head. Since 2007 DARPA urban challenge[1][2][3], Velodyne HDL-64E S2 has been widely applied in SLAM, 3D perception and other area of robotics.

Many work has been done in multi-beam LIDAR and camera calibration. In [4], author present mutual information (MI) based algorithm for automatic extrinsic calibration of a 3D laser scanner and optical camera system. [5] provides an approach for external calibration of a 3D laser scanner with an omni-directional camera system. [6] proposes a method to estimating the intrinsic parameters of a 3D LIDAR while at the same time computing its extrinsic calibration with respect to a rigidly connected camera. Some other work focuses on intrinsic parameters of multi-beam LIDAR, e.g. [7][8] use pattern planes to calibrate intrinsic parameters. [9] mentions both intrinsic parameters calibration and extrinsic parameters calibration.

As Fig 1 and Fig 2 present, a HDL64E S2 LIDAR has 64 laser emitters along the vertical direction. The angle interval between adjacent emitters is about 0.4 degree. The resolution of azimuth angle is 0.09 degree which is much higher than elevation angle's resolution. Due to the geometrical model of LIDAR system, data is sparse in vertical direction, especially at long distances. This effect makes 3D features unstable at the distance of $15 \mathrm{~m}$ or more. However, 2D features in planes which parallel to horizon plane are more stable, such as polelike features, e.g. tree trunks, lampposts of streetlights.

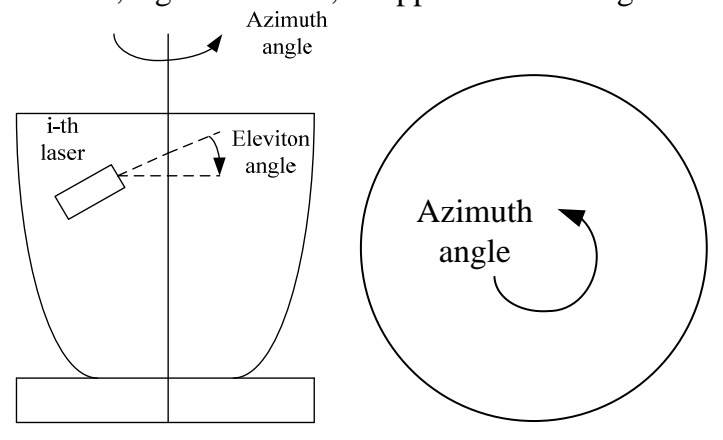

Figure 1. geomertrical model of HDL64ES2 LIDAR: left figure is sideview of the LIDAR; right figure is top view of the LIDAR

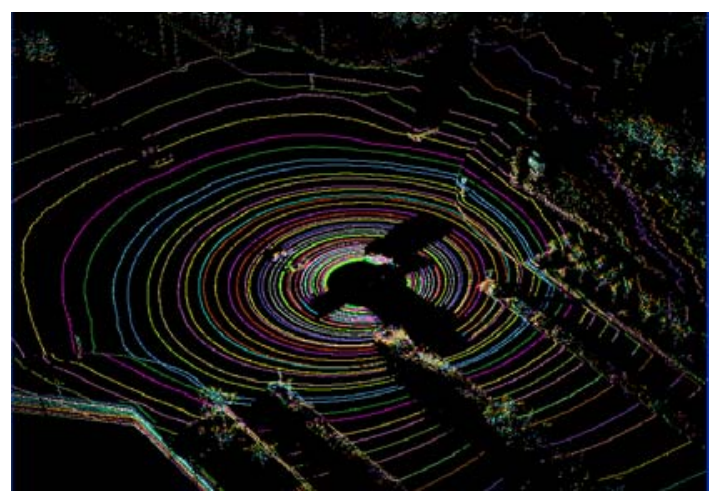

Figure 2. data assembled by HDL64ES2: different color represents different laser beam

Given that the LIDAR is often mounted on a vehicle platform which also has an IMU and GPS system, we propose a two step unsupervised calibration method: computing rotation matrix caused by pitch angle and roll angle through estimating ground plane; computing rotation matrix caused by navigation angle through matching polelike obstacles in a series of the vehicle poses gotten from the IMU and GPS system.

The paper is organized as follows: section II describes system architecture of our vehicle platform; section III 
introduces data preprocessing; section IV presents the calibration procedure; section VI provides the experiment results.

\section{SYSTEM ARCHITECTURE}

Our vehicle is shown in Fig 3. The Velodyne HDL-64E S2 LIDAR sensor is mounted on the top of the vehicle. The vehicle pose estimation system is comprised of IMU, GPS and a wheel encoder. It reliably provide ego-motion data.

The vehicle frame and the LIDAR frame are shown in Fig.4. We define yaw angle $\gamma$ rotates around Z-axis, roll angle $\beta$ rotates around $\mathrm{Y}$-axis, pitch angle $\alpha$ rotates around $\mathrm{X}$-axis.

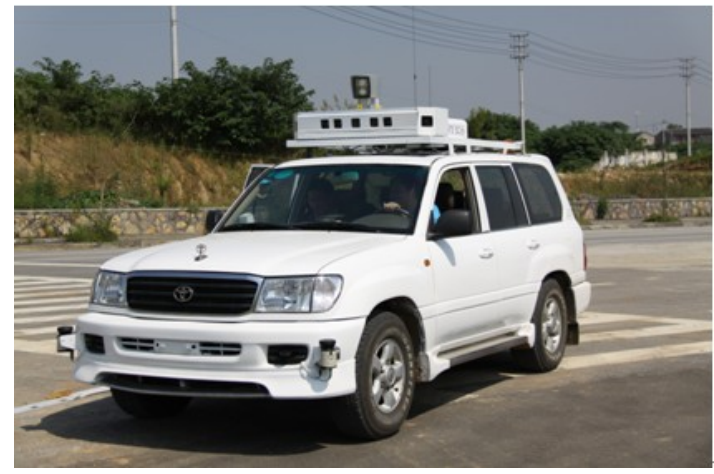

Figure 3. Vehicle platform for experiment

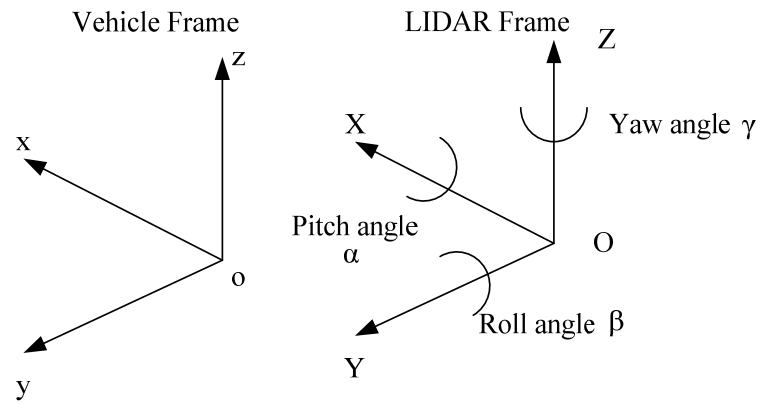

Figure 4. Vehicle frame and LIDAR frame

\section{DATA PREPROCESSING}

The original sensor readings are in spherical coordinates, while we use $3 \mathrm{D}$ points in Cartesian coordinates. So the sensor readings are transformed into Cartesian coordinates first according to LIDAR geometrical model.

Denoting the $i$ th laser beam's elevation angle with $\theta_{i}$, rotation angle in time $t$ is $\phi_{t}$, the measurement is $r_{t}$, the computation of $3 \mathrm{D}$ points is as (1):

$$
\left(\begin{array}{c}
x_{t} \\
y_{t} \\
z_{t}
\end{array}\right)=r_{t}\left(\begin{array}{c}
\cos \left(\theta_{i}\right) \cos \left(\phi_{t}\right) \\
\cos \left(\theta_{i}\right) \sin \left(\phi_{t}\right) \\
\sin (\theta)
\end{array}\right)
$$

Due to the motion of the vehicle and the fact that the scanner takes a non-negligible amount of time $(60 \mathrm{~ms} \sim 150 \mathrm{~ms})$ to complete one rotation, the point clouds will be distorted by vehicle motion. We use vehicle pose data to rectify the point clouds.

The vehicle pose information can be received at the instant of every frame ending. The pose information in one frame at time $t$ is computed by linear interpolating the poses difference between the current frame and the last frame. Denoting LIDAR working at $f \mathrm{~Hz}$, the pitch angle difference of the concessive two frames is $\alpha$. The pitch angle at time $t$ is approximated by linear interpolation as $a(t)=f \cdot t \cdot a$, the other angles and translations components can be computed analogously.

From these angles and translations, we can derive the rotation matrix and translation at time $t$.

$$
\left(\begin{array}{c}
\overline{x_{t}} \\
\overline{y_{t}} \\
\bar{z}_{t}
\end{array}\right)=R_{t}\left(\begin{array}{c}
x_{t} \\
y_{y} \\
z_{t}
\end{array}\right)+T_{t}
$$

\section{APPROACH}

According to Euler-angles' definition, LIDAR frame to vehicle frame rotation matrix $R$ and translation $T$ can be decomposed into two parts as ( 3 ) shown:

rotation $R_{1}$ caused by pitch angle $\alpha$, roll angle $\beta$ and translation $T_{1}$ caused by z component;

rotation $R_{2}$ caused by yaw angle $\gamma$ and translation $T_{2}$ caused by $\mathrm{x}$ and y component.

$$
R=R_{1} R_{2}, T=T_{1}+T_{2}
$$

\section{A. Ground extracting and $R_{1}, T_{1}$ Computation}

The vehicle is driven on a flat urban road. Ground plane is extracted by fit $3 \mathrm{D}$ points in region of interest through RANSAC. (4):

The ground plane in LIDAR frame can be represented as

$$
A x+B y-z+c=0
$$

Assuming yaw angle, $\mathrm{x}$ component and y component in translation are all zero. Sensor to vehicle's pitch angle, rotation angle and translation in $Z$ axis are computed as fig 5 . 


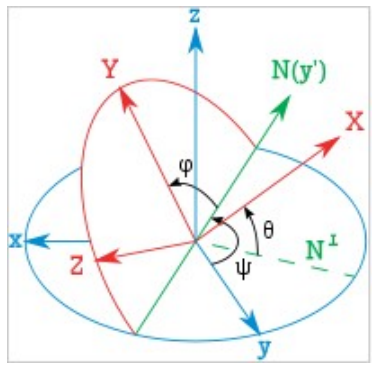

Figure 5. Tait-Bryan angles Z-Y-X

$$
\begin{gathered}
R_{1}=\left[\begin{array}{ccc}
\cos \psi & \sin \psi & 0 \\
-\sin \psi & \cos \psi & 0 \\
0 & 0 & 1
\end{array}\right]\left[\begin{array}{ccc}
1 & 0 & 0 \\
0 & \cos \theta & \sin \theta \\
0 & -\sin \theta & \cos \theta
\end{array}\right]\left[\begin{array}{ccc}
\cos \varphi & \sin \varphi & 0 \\
-\sin \varphi & \cos \varphi & 0 \\
0 & 0 & 1
\end{array}\right] \\
T_{1}=\left[\begin{array}{l}
0 \\
0 \\
C
\end{array}\right]
\end{gathered}
$$

\section{B. Pole-like Obstacle labeling and $R_{2}, T_{2}$ Computation}

Similar to [10], we slice point clouds to get arc features and use arc curvature centers as labeled points for calibration.

Two group of points $P_{i, t}, P_{i, t+1}$ are labeled features in two consecutive data frame $f_{t}, f_{t+1}$, so $P_{i, t}$ and $P_{i, t+1}$ can be matched as :

$$
R_{l, i, t} P_{i, t}+T_{l, i, t}=P_{i, t+1}
$$

At time $t+1$, we can derive vehicle local pose difference $R_{s, t}$ and $T_{s, t}$ to its pose at time $t$. For LIDAR is rigidly connected to vehicle, rotation in LIDAR frame equals to rotation $R_{s, t}$, so $R_{l, i, t}=R_{s, t}$. Translation $T_{l, t}$ of LIDAR frame at time $t$ is approximate by the average translation of all the match points at the time..

$$
T_{l, t}=\frac{1}{n} \sum_{i=1}^{n}\left(P_{i, t+1}-R_{s, t} P_{i, t}\right)
$$

The relation of $T_{l, t}, T_{s, t}$ can be used to compute rotation and translation between the two system.

$$
R_{p} T_{l, t}+T_{p}=R_{s, t} T+T_{s, t}
$$

We compute $R_{p}, T_{p}$ by solving least square formula (10)

$$
E=\sum_{t=1}^{T}\left(R_{p} T_{l, t}+T_{p}-R_{s, t} T_{p}-T_{s, t}\right)^{2}
$$

For the coefficients are linear in (10), it has the solution similar to (11), then it's easy to get $R_{2}, T_{2}$ :

$$
X=\left(A^{T} A\right)^{-1} A^{T} B
$$

$$
R_{2}=\left[\begin{array}{cc}
R_{p} & 0 \\
0 & 1
\end{array}\right] T_{2}=\left[\begin{array}{c}
T_{p} \\
0
\end{array}\right]
$$

\section{EXPERIMENT AND RESULTS}

The calibration algorithm is tested by our vehicle platform in real environment .

\section{A. Ground Extraction and Pose estimation}

The region of interest for ground estimation is set as: $[-500 \mathrm{~cm}, 800 \mathrm{~cm}]$ in $\mathrm{x}$-axis, $[-2000 \mathrm{~cm}, 3500 \mathrm{~cm}]$ in $\mathrm{y}$-axis, shows in fig.6;

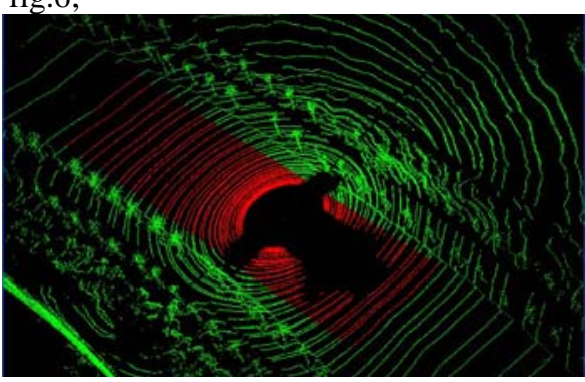

Figure 6. Ground Plane Extraction: red area is the region of interest for RANSAC

The result of RANSAC ground plane is:

$-0.0004 x+0.0041 y-z-227.3088=0$

$R_{1}, T_{1}$ are computed by (5) (6).

\section{B. Pole-like Feature Extraction and Pose Estimation}

The vehicle is driven along the curved road at speed of $10 \mathrm{~km} / \mathrm{h}$, the track is shown in fig. 7 .

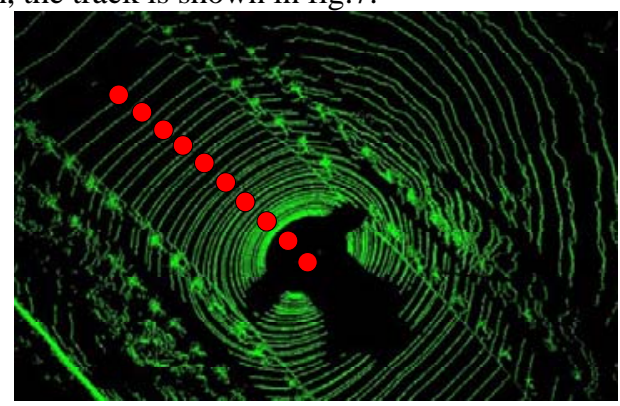

Figure 7. Vehicle’s Track

Every five frame, system extracts pole-like feature(see fig.8) and record vehicle pose from pose estimation system, Then use(8) - (12) to compute $R_{2}, T_{2}$. 


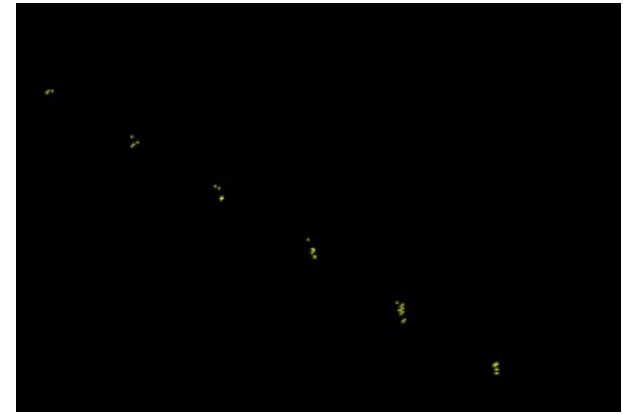

Figure 8. Pole-like Feature Extraction

\section{Performance Evaluation}

We use supervised calibration which takes a lot of hand measurements as ground truth. After our unsupervised calibration, the error distances are dropping significantly. In fig.9, after calibration ground plane in LIDAR frame is very close to the ground in vehicle frame, most of error distances under $5 \mathrm{~cm}$; in fig.10 calibration also reduces 3D points' error distance between LIDAR frame and vehicle frame.

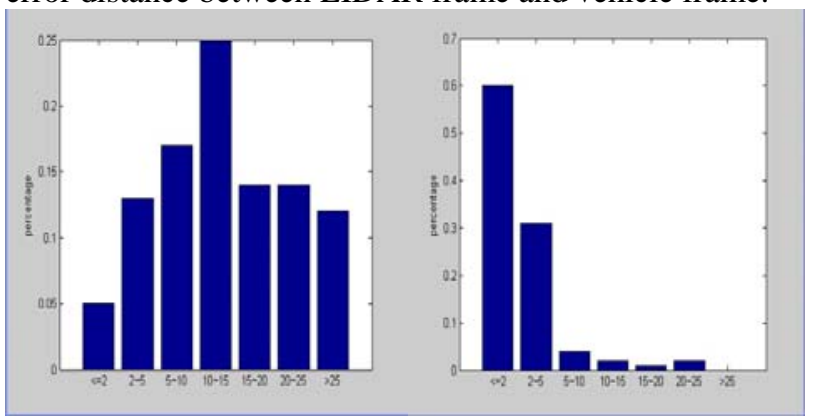

Figure 9. Error distance to ground plane comparison

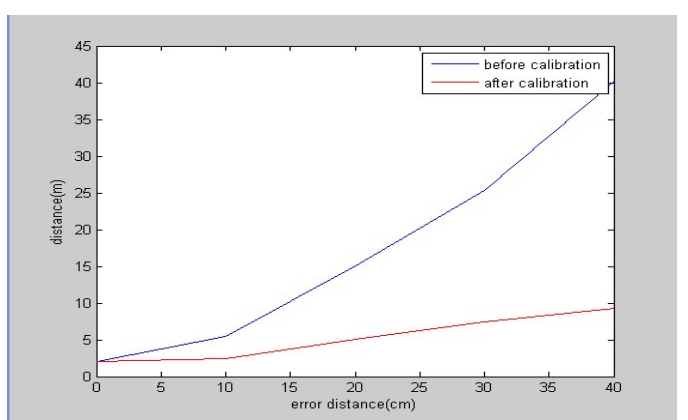

Figure 10. Error distance to 3D points comparison

\section{CONCLUSION}

Multi-beam LIDAR's importance is increasing in the field of robotics. The powerful ability of 3D perception makes it irreplaceable in 3D-SLAM. It's sure that there are still many of application can be developed in the huge amount of data provided by multi-beam LIDAR. For any sensor, calibration is one of the most important procedures before it generates valid data. The paper has presented an unsupervised method which shows how to simply and efficiently do calibration. We now aim to work on the calibration of LIDAR intrinsic parameters, possibly simultaneous calibration of LIDAR intrinsic parameters and extrinsic parameters.

\section{ACKNOWLEDGMENT}

The presented work has been done within in the scope of the "Visual and Acoustic Cognitive Computing Intelligence Vehicle Platform". The project is funded by the National Natural Science Fund (Code: NFSC NO. 90820306)

\section{REFERENCES}

[1] Yoon, J. Y. Crane, C. D.] LADAR based Obstacle Detection in an Urban Environment and its Application in the DARPA Urban Challenge International Conference on Control, Automation and Systems, Vols 1-4,2008

[2] Toth, C, Grejner-Brzezinska, D. A.Ozguner, U. From the DARPA Grand to Urban Challenge: Mobile Mapping Supporting Autonomous Vehicle Navigation Proceedings of the 2007 National Technical Meeting of the Institute of Navigation 2007

[3] Hurdus, J., Bacha, A.Bauman, C. VictorTango Architecture for Autonomous Navigation in the DARPA Urban Challenge Journal of Aerospace Computing Information and Communication

[4] G. Pandey, J. R. Mcbride, S. Savarese, and R. M. Eustice, "Automatic Targetless Extrinsic Calibration of a 3D Lidar and Camera by Maximizing Mutual Information,” Twenty-Sixth AAAI . . . , vol. 26, pp. 2053-2059, 2012.

[5] Pandey G, McBride J, Savarese S and Eustice R (2010) Extrinsic calibration of a 3D laser scanner and an omnidirectional camera. In 7th IFAC Symposium on Intelligent Autonomous Vehicles.

[6] Mirzaei, F.M.; Kottas, D.G.; Roumeliotis S.I. 3D Lidar-camera intrinsic and extrinsic calibration: Identifiability and analytical leastsquares-based initialization. Int. J. Robot. Res. 2012, 31, 452-467.

[7] Atanacio-Jimenez,G.; Gonzales-Barbosa, J.J; Hurtado-Ramos, J.B.; Francisco, J.; Jimenez-Hernarndes,H.; Garcia-ramirez T.; Velodyne HDL-64E calibration using pattern planes. Int, J. Adv. Robotic. Syst.2011,8, 70-82.

[8] Muhammad, N.; Lacroix, S. Calibration of a Rotating Multi-Beam Lidar. In Proceedings of the IEEE/RSJ International Conference on Intelligent Robots and Systems (IROS), Toulouse, France, 18-22 October 2010; pp. 5648-5653.

[9] J. Levinson and S. Thrun, "Unsupervised Calibration for MultibeamLasers," in International Symposium on Experimental Robotics, 2010.

[10] Luo, D.; Wang, Y. Rapid Extracting Pillars by Slicing Point Clouds. Int. Arch. Photogramm. Remote Sens. 2008, XXXVII-B3b, 215-218. 